\title{
Reply: Comment on 'MicroRNA-214 suppresses growth, migration and invasion through a novel target, high mobility group AT-hook 1, in human cervical and colorectal cancer cells'
}

\author{
Karthik Subramanian Chandrasekaran ${ }^{1}$, Anusha Sathyanarayanan ${ }^{1}$ and Devarajan Karunagaran *,1
}

${ }^{1}$ Department of Biotechnology, Bhupat and Jyoti Mehta School of Biosciences, Indian Institute of Technology Madras, Chennai, Tamil Nadu 600036, India

Sir,

The authors would like to acknowledge the time and effort spent in critical assessment of their work (Chandrasekaran et al, 2016) by Cristóbal et al. Since they have pointed out that the effect on the HMGA1 protein expression is minimal, the authors draw their attention to these data:

1. The ectopic expression of miR-214 reduced mRNA levels of HMGA1 by $15-30 \%$ (Figure 2B) and HMGA1 $3^{\prime}$ UTR-regulated luciferase activity by $25-33 \%$ (Figure 2E) in SW480 and SW620 cells, respectively.

2. The effect of ectopic expression of miR-214 on HMGA1 protein levels in these cell lines is quite consistent and reproducible.

3. Suppressing the endogenous expression of $m i R-214$ has profound effect on HMGA1 expression at the protein level (increased HMGA1 by $40-70 \%$ ) in both these cell lines (Figure 5A). This clearly points out the ability of endogenous $m i R-214$ to suppress $H M G A 1$ expression.

These reasons are enough to claim that miR-214 targets HMGA1 and downregulates its expression. The complexity involved in several miRNAs regulating the expression of a single target spatially and temporally has been discussed in the paper and stated in the query itself. Decoding the entire regulatory miRNA network of HMGA1 was not within the scope of this study.

Cristóbal et al have pointed out that the anti-tumourigenic effects of miR-214 might be mediated through the $\mathrm{Wnt} / \beta$-catenin pathway and not by $H M G A 1$. Once again, attention is drawn to these:

1. The effects of ectopic expression of $m i R-214$ (Figures $3 \mathrm{~A}-\mathrm{C}$ ) and suppression of expression of HMGA1 (Figures $4 \mathrm{~A}-\mathrm{C}$ ) on proliferation, migration and invasion of SW480 and SW620 cells are very much comparable.

2. The effects of ectopically co-expressing $m i R-214$ and HMGA1 (as suggested in the query) were addressed (Figure 6), and the conclusions are not likely to change by ectopically expressing $m i R-214$ or suppressing HMGAl expression together.

3. The reference cited to show the ability of HMGA1 to positively regulate the Wnt- $\beta$-catenin pathway in the query also suggests that $m i R-214$ could directly suppress $\beta$-catenin, downregulating the Wnt pathway through negative regulation of HMGA1. In addition, this cannot be directly extended to CRC since the referred studies were conducted in hepatic cancer cells and will need experimental validation in CRC cells, which are known to exhibit constitutive Wnt signalling.

In summary, since miR-214 does indeed target HMGA1 and downregulates its expression, and since HMGA1 certainly behaves as an oncogene in CRC cells, part of the tumour-suppressive functions of miR-214 may occur through suppression of HMGA1 expression. The functional relevance of $m i R-214$ targeting HMGA1 could be inferred from the fact that $m i R-214$ is still able to exert its tumour-suppressing function in the presence of ectopically expressed HMGA1.

Further, the study focused on ascertaining whether an inverse correlation exists between the expression levels of miR-214 and HMGA1. Since a study on 20 pairs (normal and tumour) of tissues could be considered insufficient to obtain conclusiveness, a much larger population (322 samples from TCGA) was analysed; the results from both the analyses reinforced each other and evidently showed the inverse correlation in the expression levels of miR-214 and HMGA1 (Figure 1).

The authors agree with Cristóbal et al that protein-mRNA correlation (with respect to $H M G A 1$ expression) would help in concluding about the status of HMGA1 in CRC and would strengthen the conclusions. The contradiction observed in HMGA1 status in CRC tissues (Liang et al, 2013) could be attributed to differences in the genetic make-up of the populations studied, and their results are contraindicated by the data on HMGA1 expression in CRC obtained from TCGA. In conclusion, the authors would like to reiterate that the study does not discount the roles that may be played by other miRNAs that may target HMGA1 or other targets of $m i R-214$, and this is discussed in the paper. This is why, in quite a few relevant instances, it is asserted that $m i R-214$, in part, suppresses growth and motility in cancer cells by downregulating HMGA1, as it is intuitively recognised that further studies are most certainly required to unravel the outcome of a miRNA-regulatory network on the behaviour of HMGA1 in CRC and other cancers.

\section{CONFLICT OF INTEREST}

The authors declare no conflict of interest.

\section{REFERENCES}

Chandrasekaran KS, Sathyanarayanan A, Karunagaran D (2016) MicroRNA-214 suppresses growth, migration and invasion through a novel target, high mobility group AT-hook 1, in human cervical and colorectal cancer cells. Br J Cancer 115: 741-751.

Liang L, Li X, Zhang X, Lv Z, He G, Zhao W, Ren X, Li Y, Bian X, Liao W, Yang G, Ding Y (2013) MicroRNA-137, an HMGA1 target, suppresses colorectal cancer cell invasion and metastasis in mice by directly targeting FMNL2. Gastroenterology 144: 624-635.e4.

This work is published under the BJC's standard license to publish agreement After 12 months the license terms will change to a Creative Commons AttributionNonCommercial-Share Alike 4.0 Unported License. 